\title{
Responsabilidad social, un estudio bibliométrico
}

\section{Social responsibility, a bibliometric analysis}

\author{
Jorge Alejandro Sánchez Henríquez ${ }^{1}$, Ignacio Andrés Yáñez Vidal ${ }^{2}$
}

\begin{abstract}
RESUMEN
El propósito de este artículo es presentar un análisis bibliométrico de la producción científica sobre Responsabilidad Social. Los artículos fueron procesados con MSExcel, Bibliometrix y VosViewer. La producción se concentró en negocios, administración, contabilidad, ciencias sociales y economía, el tema más recurrente fue el rendimiento financiero y la productividad, el desarrollo conceptual se concentró en pocas revistas, la mayoría de la productividad se asoció a solo tres autores principales, los cuales tienen casi la totalidad de las publicaciones y concentran la mayor cantidad de citaciones. Esto se replicó a nivel de continentes y países, lo cual se debería revertir en el futuro.
\end{abstract}

Palabras claves: bibliometría, responsabilidad social, responsabilidad social empresarial.

Recepción: 06/09/2021. Aprobación: 09/11/2021

\begin{abstract}
The purpose of this article is to carry out a bibliometric analysis of the scientific research on Social Responsibility. The articles analysed were processed using the MSExcel, Bibliometrix and VosViewer tools. The scientific research was concentrated on business, administration, accountability, social sciences, and economy. The most recurrent topics were financial performance and productivity. Conceptual development was concentrated in a few journals, with most of the research being associated with three main authors, who have written almost all the articles and who have been quoted the most. This was replicated in the analysis by countries and continents, which should be reversed in the future.
\end{abstract}

Keywords: bibliometrics, social responsibility, corporate social responsibility.

1 Universidad de Talca, Facultad de Economía y Negocios, Talca, Chile, jsanchez@utalca.cl

2 Universidad de Talca, Facultad de Economía y Negocios, Talca, Chile, ignacio.andresyv@gmail.com 


\section{INTRODUCCIÓN}

Para conocer el estado del arte en una disciplina o tema, hay que aplicar un método y según Castellanos, Fúquene, y Ramírez (2011) uno de los mejores métodos de búsqueda para analizar la evolución del conocimiento científico es la bibliometría, que ayuda a comprender los cambios en el conocimiento científico sobre un tema, en este contexto la bibliometría es utilizada en diferentes áreas y ambientes, según Arbeláez y Onrubia (2016), se aplica en bases de datos y revistas científicas o académicas.

Desde el punto de vista de estudios bibliométricos, se constató que la Responsabilidad Social ha sido poco estudiada integralmente y destacan los trabajos de Aguinis y Glavas (2012) en la plataforma Scopus y los aportes de Duque y Cervantes (2019) en Web of Science.

Para la Responsabilidad Social destaca el estudio de Bandura (2001), sobre la teoría cognitiva distinguiendo tres modos de agencia, tales como agencia personal directa, agencia proxy y agencia colectiva, otorgando prioridad a la eficacia colectiva para ejercer control sobre los destinos personales. También se menciona a Porter y Kramer (2006), quienes muestran una forma particular de ver la relación entre las empresas y la sociedad introduciendo un marco para que las empresas identifiquen las consecuencias de sus acciones, oportunidades de beneficio y la determinación de iniciativas de Responsabilidad Social Empresarial.

El objetivo de la investigación fue realizar un análisis de la producción científica sobre Responsabilidad Social en revistas reconocidas a nivel global. La investigación se basó en un análisis bibliométrico de artículos sobre el tema de Responsabilidad Social, publicados en revistas indexadas en las bases de datos Scopus y Web of Science, según Houston y Delevan (1990) y Stallings y Ferris (1988), la investigación científica se difunde a la comunidad a través de revistas indexadas, en efecto, según Perry y Kraemer (1986), la calidad de la producción científica se mide en las revistas indexadas en bases de datos bibliográficas, en definitiva, las revistas son consideradas por Legge Jr y Devore (1987) como un parámetro pertinente y un excelente indicador, para evaluar la calidad de la producción de líneas de investigación científica.

La bibliometría se ha utilizado en distintas áreas del conocimiento científico, en educación con Salah, Rahim, y Carretero (2013), en innovación con Pineda (2015), en servicios de internet con Duque, Cervera, y Rodríguez (2006), en sostenibilidad con Janik, Ryszko, y Szafraniec (2020), en salud con Espinoza, Lioo y Villanueva (2018), en turismo con Tomazzoni y Tavares (2014), en ética personal y liderazgo con Correa, Rodríguez, y Pantoja (2018), en ciencias básicas con Anchondo, Tarango, y Ascensio (2014), en clima organizacional con Pedraza (2020), en productividad con Maturana y Andrade (2019), en auditoría con Behrend y Eulerich (2019) y Sánchez Henríquez y Yáñez Vidal (2021), en recursos humanos con Weng et al. (2018), en finanzas con Bernal (2013), en género y equidad con Holman, Stuart y Hauser (2018).

La relevancia y pertinencia de realizar estudios bibliométricos sobre responsabilidad empresarial, está absolutamente vigente y alienta la utilización de la bibliometría en este tipo de estudios (De Bakker, Groenewegen y Den Hond 2005; Duque y Cervantes 2019). 
La metodología de búsqueda fue testeada y probada inicialmente, en la base de datos SciELO y Web of Science que tenían una gran cantidad de revistas indexadas y miles de artículos. La información fue extraída en formato CSV en Scopus y en formato de texto plano para Web of Science. Posteriormente se ingresaron a la herramienta Bibliometrix y a VosViewer. El análisis de los datos incluyó evaluaciones de la producción científica por año, fuentes, autores, temas, palabras claves, ranking y citas a nivel de revistas. Finalmente se analizaron, en detalle, los artículos, que estaban directamente relacionados con Responsabilidad Social.

\section{Marco Teórico}

Para Nechi, Aouni, y Mrabet (2020) la Responsabilidad Social persigue el desarrollo económico y trata de mejorar las condiciones sociales de las sociedades humanas. Según Urdaneta et al. (2016) esta visión comienza en Estados Unidos, como consecuencia de conflictos bélicos en la década 50, las organizaciones deben trabajar en estrategias que logren diseñar empresas sustentables con la sociedad y su ambiente (Williamson 2005).

La Responsabilidad Social es un instrumento muy útil para lograr las metas que las organizaciones persiguen, para Madero (2020) y Peloza y Shang (2011), la Responsabilidad Social le ayuda a las organizaciones a cumplir con los objetivos planteados, y debe ser incluida en la estrategia, según Sardana et al. (2020) todas las empresas deberían interesarse en implementar Responsabilidad Social en sus estrategias, varios autores afirman que a través de ella la organización logrará obtener ventajas competitivas (Larrán y Andrades 2015). Una visión complementaria que siempre debería incluir la estrategia es la perspectiva planteada por Salazar, Hidalgo, y Manríquez (2017) quienes sostienen que el nivel de Responsabilidad Social que alcancen las organizaciones depende en gran medida de su capital humano, esto es avalado por varios autores, entre los cuales están Fernández, Acosta, y López (2020). Se deben complementar e integrar las políticas y prácticas de recursos humanos y Responsabilidad Social, para lograr la sostenibilidad de la organización, solo a través de personal comprometido y satisfecho se puede obtener rentabilidad y lograr crecimiento del negocio (Macke y Genari 2019).

La relevancia y amplitud conceptual de la Responsabilidad Social queda de manifiesto en trabajos clásicos como los de Donabedian (1988), Porter y Kramer (2006) y Waddock y Graves (1997) y absolutamente refrendada en investigaciones contemporáneas como las de Franco et al. (2020) y Javed et al. (2020).

La Responsabilidad Social, es un concepto absolutamente multifactorial, integral y muy complejo, esta mirada la sostienen varios autores que se aproximan al concepto a través de diversas dimensiones, variables, teorías y modelos (Bai y Chang 2015 y Fernández 2009).

Al revisar la producción científica y ver la evolución que ha tenido el concepto una mirada evolutiva bien trabajada es la planteada por Barbosa, Medina y Vargas (2014) y complementada por Severino (2017), a través del tiempo se puede observar que se han definido diferentes perspectivas y tipos de responsabilidad, es así como se habla de Responsabilidad Social Empresarial RSE (Organizacional o Corporativa), Responsabilidad Social Gubernamental (RSG), Responsa- 
bilidad Social Ambiental (RSA), Responsabilidad Social Universitaria (RSU) y Responsabilidad Social Individual (RSI) (Dahlsrud 2008; Madero 2020; Romero y Lara 2016; Rosolen y Maclennan 2016; Tello y Rodríguez 2014).

Las empresas esgrimen múltiples motivaciones, por las cuales ellas se interesan en la Responsabilidad Empresarial (Saha et al. 2020; Yang, Colvin, y Wong 2013). Las organizaciones tratan de implementar políticas de Responsabilidad Social, pero ello no resulta para nada fácil, ya que implica un profundo cambio organizacional (Madero 2020), el cambio implica adoptar nuevas políticas y prácticas de gestión, para después involucrar a todos sus grupos de interés (Arnaud y Wasieleski 2014; Becker y Smidt 2016). Lo que queda absolutamente claro es que la Responsabilidad Social, es un tema muy relevante para la permanencia y desarrollo de la organización actual y que cada día serán más, los que las incluyan e implementen en sus estrategias de negocio.

\section{METODOLOGÍA, MATERIALES Y METODOS}

Para la elaboración de este artículo se realizó un estudio bibliométrico descriptivo a la producción científica referente a Responsabilidad Social, con la finalidad de recoger información de manera independiente o conjunta sobre los conceptos o las variables a las que se refieren (Hernández Sampieri 2014). La búsqueda se desarrolló en la base de datos Scopus (Elsevier), la que actualmente cuenta con más de 23.000 revistas indexadas activas. Según Houston y Delevan (1990) y Duque y Cervantes (2019), la investigación científica se difunde a la comunidad a través de revistas indexadas, en efecto, según Perry y Kraemer (1986), la calidad de la producción científica se mide en las revistas indexadas en bases de datos bibliográficas, en definitiva, las revistas son consideradas por Legge Jr y Devore (1987) y Belter (2015) como un parámetro pertinente y un excelente indicador, para evaluar la calidad de la producción de líneas de investigación científica.

La investigación realizada comenzó con una revisión acabada del estado del arte en los temas de bibliometría y Responsabilidad Social, con el objetivo final de construir un marco teórico adecuado al objetivo de los investigadores, para Gaede y Rowlands (2018) es una excelente forma de combinar y concentrar una gran cantidad de investigaciones producto de las cuales todo lo que se encuentre se puede proyectar al futuro. Posteriormente se comienza a trabajar directamente con los artículos de las bases de datos Scopus, para ello se construyeron varias ecuaciones de búsqueda, partiendo de una lo más amplia posible, para cubrir la mayor cantidad de documentos, la cual posteriormente se fue ajustando para ir filtrando de mejor manera los documentos encontrados.

Con respecto a la ecuación de búsqueda, esta se centró en los principales 5 tipos de Responsabilidad Social propuestos por Romero y Lara (2016) los cuales son Responsabilidad Social Empresarial o Corporativa (RSE), Responsabilidad Social Universitaria (RSU), Responsabilidad Social Ambiental (RSA), Responsabilidad Social Individual (RSI) y Responsabilidad Social Gubernamental (RSG). Esta metodología fue testeada y probada previamente, en las bases de datos Web of Science y SciELO que a la fecha cuenta con más de 10.000 y 1.700 revistas indexadas respectivamente. De la búsqueda realizada en los sitios web de la base de datos Scopus se obtuvo un total final de 14.347 resultados en lo que se refiere a la producción científica sobre el concepto de Responsabilidad Social. 
Una vez ingresada la ecuación de búsqueda en la base de datos, se extrajeron las referencias bibliográficas de los resultados encontrados en formato CSV (valores separados por comas). Posteriormente se ingresan a la herramienta Bibliometrix, mediante el software RStudio y posteriormente a VosViewer. Los resultados obtenidos fueron sujetos a un análisis bibliométrico inicial mediante la herramienta MS Excel, con el objetivo de medir la producción científica anual, se midió también la producción por países, por continentes, por temas, por palabras claves, por ranking y áreas temáticas que abarcaban los documentos.

Los documentos extraídos, dan cuenta de toda la producción científica en la base de datos Scopus, en efecto se cubrió el período que comenzaba desde el año 1964 al año 2020, por tanto, el trabajo consideró la totalidad de artículos vigentes en ambas bases, lo cual reflejó toda la producción científica en Responsabilidad Social.

\section{RESULTADOS}

La producción científica relacionada con la Responsabilidad Social en la base de datos Scopus data del año 1964 (Figura 1), reflejando hasta el año 2003 su período de menor productividad, arrojando un promedio de 9 documentos publicados por año. En los 11 años posteriores, es decir, hasta el año 2014 la producción científica sobre Responsabilidad Social registra un considerable aumento, lo anterior debido a que el promedio de publicación de artículos por año es de 496. En adelante hasta el año 2020 se registra un aumento aún más notorio, obteniendo un promedio de publicación anual de 1.505 documentos por año, por lo tanto, entre los años 2015 y 2020 se registra el periodo de mayor productividad.

\section{Figura 1}

Producción científica anual sobre Responsabilidad Social en Scopus, período 1964 - 2020.

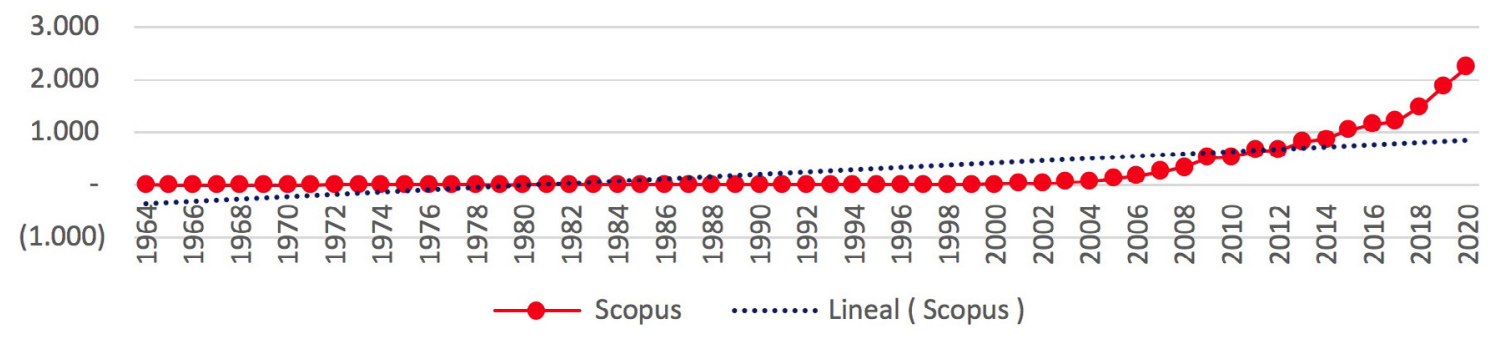

Fuente: Elaboración propia con base en datos de Scopus (2021).

Se constató que una gran cantidad de países realizan publicaciones sobre el tópico de Responsabilidad Social en general, destacando fuertemente la presencia de 4 continentes. En primer lugar, el continente americano se posiciona como el mayor productor de documentos de esta índole, siendo Estados Unidos el país que cuenta con la mayor cantidad de publicaciones, tanto a nivel continental como a nivel mundial con 3.036 documentos aproximadamente. En lo que respecta al continente europeo, se ve representado fuertemente por Reino Unido y España, en la misma línea también se destaca a China como el país asiático que más contribuciones realiza. Finalmente se 
encuentra el continente de Oceanía, en el cual Australia se posiciona como el mayor productor de artículos científicos sobre Responsabilidad Social.

En cuanto a la distribución de la producción científica por área temática (Tabla 1), se pudo observar que las áreas de negocios, administración y contabilidad, ciencias sociales, economía y finanzas, ciencias del medioambiente, artes y humanidades y energía, son las que predominan, ya que en su conjunto tienen 26.951 documentos que se encasillan en al menos una de estas categorías, lo que significa un $87 \%$ de la producción científica analizada, para efectos del análisis por área temática.

Tabla 1

Distribución por área temática en Scopus, período 1964 - 2020.

\begin{tabular}{lcccccc}
\hline \multicolumn{1}{c}{ Área } & RSE & RSU & RSI & RSA & RSG & Total \\
\hline Business, Management and Accounting & 9.822 & 37 & 8 & 12 & 3 & 9.882 \\
Social Sciences & 6.876 & 103 & 10 & 9 & 7 & 7.005 \\
Economics, Econometrics and Finance & 4.118 & 15 & 3 & 6 & 2 & 4.144 \\
Environmental Science & 2.783 & 16 & 2 & 7 & 2 & 2.810 \\
Arts and Humanities & 1.723 & 27 & 6 & 4 & - & 1.760 \\
Energy & 1.333 & 13 & - & 4 & - & 1.350 \\
Engineering & 973 & 5 & 2 & 3 & 1 & 984 \\
Decision Sciences & 685 & 7 & - & 1 & - & 693 \\
Computer Science & 420 & 4 & 1 & 2 & - & 427 \\
Medicine & 359 & 6 & 3 & 1 & 1 & 370 \\
Psychology & 355 & 9 & 6 & - & - & 370 \\
Otros & 1.054 & 6 & 5 & 3 & 1 & 1.069 \\
\hline Total & 30.501 & 248 & 46 & 52 & 17 & 30.864 \\
\hline
\end{tabular}

Fuente: Elaboración propia con base en datos de Scopus (2021).

La RSE se perfila como el tipo de Responsabilidad Social más relevante, ya que por sí solo, registra una proporción de un $98,9 \%$ de los resultados encontrados, seguido por la RSU 0,8\% de la producción científica, mientras que la RSI, RSA y RSG en su conjunto contribuyen con un 0,3\%. Lo anterior consolida a la RSE como el tipo de Responsabilidad Social que cuenta con un mayor nivel de desarrollo en la base de datos Scopus.

Posteriormente se realizó un análisis a las revistas que son más relevantes dentro de la producción científica en la base de datos Scopus, en lo que respecta a la Responsabilidad Social. Entre las 
fuentes más relevantes se destacan las contribuciones de la revista "Journal Of Business Ethics", la cual tiene como principales temáticas aspectos éticos en el contexto del desarrollo de los negocios, incluyendo sistemas de producción marketing, contabilidad social y económica, comportamiento organizacional y relaciones laborales. Dicha revista posee publicaciones de la RSE, RSI y RSA, además, cuenta con el puntaje más alto en lo que respecta al Citescore, la cual es una métrica que proporciona un valor adicional para efectos de comparar las revistas científicas. Este indicador refleja el promedio anual de citas recibida por una revista en los últimos 4 años, para el cual la revista "Journal Of Business Ethics" posee un puntaje de 7 al año 2019, siendo el más alto frente a sus pares en lo que respecta a la producción científica sobre Responsabilidad Social. De la misma manera la revista cuenta con el mayor puntaje en el índice SJR, el cual considera el total de citas recibidas ponderando la importancia de las revistas de las cuales fueron efectuadas dichas citas, es decir, este índice tiene como objetivo realzar la calidad de las publicaciones científicas, en esta métrica, la revista en cuestión posee un puntaje de 1,972 al año 2019, sumando además el hecho de que lidera las cifras en el indicador SNIP, con un puntaje de 2,7 al año 2019. Este indicador compara las publicaciones dentro de su área temática considerando la frecuencia con la que los autores citan a otros documentos y la inmediatez del impacto de la cita (Tabla 2).

\section{Tabla 2}

Productividad de revistas por tipo de R.S. en Scopus, período 1964 - 2020.

\begin{tabular}{|c|c|c|c|c|c|c|}
\hline $\begin{array}{l}\text { Área } \\
\text { R. S. }\end{array}$ & Revista & $\mathrm{n}$ & $\begin{array}{c}\text { Citescore } \\
2019\end{array}$ & $\begin{array}{l}\text { SJR } \\
2019\end{array}$ & $\begin{array}{l}\text { SNIP } \\
2019\end{array}$ & $\begin{array}{l}\text { Citas } \\
2020\end{array}$ \\
\hline \multirow{3}{*}{ RSE } & Journal Of Business Ethics & 1.127 & 7 & 1,972 & 2,7 & 47.630 \\
\hline & Sustainability Switzerland & 756 & 3,2 & 0,581 & 1,165 & 77.410 \\
\hline & $\begin{array}{l}\text { Corporate Social Responsibility } \\
\text { and Environmental Management }\end{array}$ & 516 & 5,9 & 0,974 & 1,625 & 6.578 \\
\hline \multirow{3}{*}{ RSU } & Opcion & 14 & 0,5 & 0,202 & 0,547 & 918 \\
\hline & Sustainability Switzerland & 10 & 3,2 & 0,581 & 1,165 & 77.410 \\
\hline & Revista Venezolana De Gerencia & 7 & 0,4 & 0,165 & 0,531 & 283 \\
\hline \multirow{3}{*}{ RSI } & Journal Of Business Ethics & 3 & 7 & 1,972 & 2,7 & 47.630 \\
\hline & Journal Of Human Values & 2 & 1,1 & 0,181 & 1,136 & 178 \\
\hline & Economic Affairs & 1 & 1 & 0,311 & 0,485 & 284 \\
\hline \multirow{3}{*}{ RSA } & Sustainability Switzerland & 3 & 3,2 & 0,581 & 1,165 & 77.410 \\
\hline & Journal Of Business Ethics & 2 & 7 & 1,972 & 2,7 & 47.630 \\
\hline & Chinese Economy & 1 & 1,1 & 0,279 & 0,948 & 411 \\
\hline \multirow{3}{*}{ RSG } & Extractive Industries And Society & 1 & 4,3 & 1,127 & 1,414 & 2.369 \\
\hline & International Review of Public Administration & 1 & 0,8 & 0,172 & 0,340 & 324 \\
\hline & Investigaciones Regionales & 1 & 1,2 & 0,360 & 0,755 & 333 \\
\hline
\end{tabular}

Fuente: Elaboración propia con base en datos de Scopus (2021). 
También se destaca la contribución de la revista "Sustainability", la cual es una revista de acceso abierto en la que sus principales temáticas radican en sostenibilidad ambiental, económica, cultural y social de los seres humanos. Esta revista registra el mayor nivel de citas recibidas sumando un total de 77.410 citas durante el año 2020, también posee publicaciones en el área de la RSE, RSA y RSU. En este último tipo de Responsabilidad Social, también se destaca la contribución de la revista "Opción", la cual posee el mayor índice de publicación en el área de la RSU aportando 14 documentos. Dicha revista es de acceso abierto y sus principales temáticas radican en las ciencias sociales y humanas con énfasis en la antropología, filosofía, lingüística, problemas del desarrollo, ciencia y tecnología. En lo que respecta a la RSG se destaca la contribución de la revista "Extractive Industries And Society", la cual tiene como objetivo difundir análisis de los impactos socioeconómicos y ambientales de la minería y de la producción de petróleo y gas.

A nivel de productividad por autores, en el área de la RSE Isabel García Sánchez se perfila como la autora más relevante con respecto a la cantidad de documentos que aporta para la búsqueda sobre Responsabilidad Social en la base de datos Scopus, proporcionando un total de 50 artículos, siendo, además, la autora que más publicaciones realiza a nivel general (Tabla 3). Las temáticas en las que se desenvuelve dicha autora radican en sostenibilidad, Gobiernos Corporativos, desempeño social corporativo, entre otros.

Tabla 3

Productividad de autores por tipo de R.S. en Scopus, período 1964 - 2020.

\begin{tabular}{clcc}
\hline Área R. S. & \multicolumn{1}{c}{ Autor } & n & Índice H \\
\hline \multirow{3}{*}{ RSE } & Kolk, Ans & 25 & 48 \\
& Vanclay, Frank & 24 & 40 \\
& García-Sánchez, Isabel María & 50 & 37 \\
\hline \multirow{3}{*}{ RSU } & Kouatli, Issam & 2 & 8 \\
& Latif, Khawaja Fawad & 2 & 8 \\
& Lanero Carrizo, Ana & 2 & 7 \\
\hline \multirow{3}{*}{ RSI } & Kim, Jae-kyeong & 1 & 23 \\
& Andereck, Kathleen L. & 1 & 22 \\
& Cheng, Julian Ming Sung & 1 & 19 \\
\hline \multirow{3}{*}{ RSA } & Sukoco, Badri Munir & 1 & 10 \\
& Özen, Sukru & 1 & 7 \\
& Dagilienė, Lina & 1 & 5 \\
\hline \multirow{3}{*}{ RSG } & Vigoda-Gadot, Eran & 1 & 34 \\
& Rasinski, Kenneth A. & 1 & 28 \\
& Smith, Tom W. & 1 & 27 \\
\hline
\end{tabular}

Fuente: Elaboración propia con base en datos de Scopus (2021). 
Es importante mencionar la contribución de Ans Kolk, quien aporta 25 documentos a la producción científica relacionada con la Responsabilidad Social, destacando además que tiene el puntaje más alto en lo que respecta al índice $h$, el cual es una métrica que equilibra el número de citas recibidas por artículo en una revista, relacionándolo con toda su producción científica, donde el volumen de citas es menor o igual al número de orden del artículo en una revisión descendente de cita (Hirsch 2005; Scimago y Citas 2006). El autor en cuestión posee una puntuación de 48 en lo que respecta al índice h y las principales temáticas en las que desarrolla su investigación científica son desempeño social corporativo, filantropía corporativa y auto eficiencia.

Por el lado de la RSU se destacan las contribuciones de Issam Kouatli, quien enmarca sus investigaciones principalmente en las áreas de toma de decisiones grupales, presupuesto de capital y fractografía y Latif Khawaja Fawad, quien investiga principalmente en las áreas referentes a comités de auditoría, gobiernos corporativos, desempeño social corporativo, modelo mental compartido, entre otros. Ambos autores cuentan con un índice h de 8.

En el área de la RSI Kim Jae-kyeong, quien cuenta con un índice h de 23, desarrolla sus investigaciones en las temáticas de reconocimiento de emociones, discriminación, racismo y retroalimentación implícita, mientras que su par Sukoco Badri Munir en el área de la RSI, cuenta con un índice h de 10 en investigaciones que radican principalmente en temas de innovación, gobernanza racional, satisfacción del consumidor, entre otros. Finalmente, por el lado de la RSG, Eran Vigoda-Gadot con un índice h de 34, siendo uno de los autores con mayor puntuación en esta métrica, sus investigaciones se desarrollaron principalmente en las áreas de habilidad política, política organizacional, capital psicológico y trabajo emocional.

A nivel de análisis por palabras claves dentro de la producción científica, se pudo apreciar las distintas temáticas que se abordan dentro de cada uno de los tipos de Responsabilidad Social en estudio, frente a lo cual se exportan las referencias desde la base de datos Scopus al software VosViewer, de manera que este último software cree un mapa con las coincidencias de palabras claves presentes en la producción científica.

En lo que respecta a la RSE (Figura 2), se pudo apreciar que los contenidos que se abordan en este tema radican principalmente en los conceptos de rendimiento financiero, comportamiento de los consumidores, sustentabilidad corporativa y en menor medida los conceptos de satisfacción laboral, rendimiento corporativo e inversión ética. 


\section{Figura 2}

Tópicos abordados sobre la RSE en Scopus, período 1964-2020.

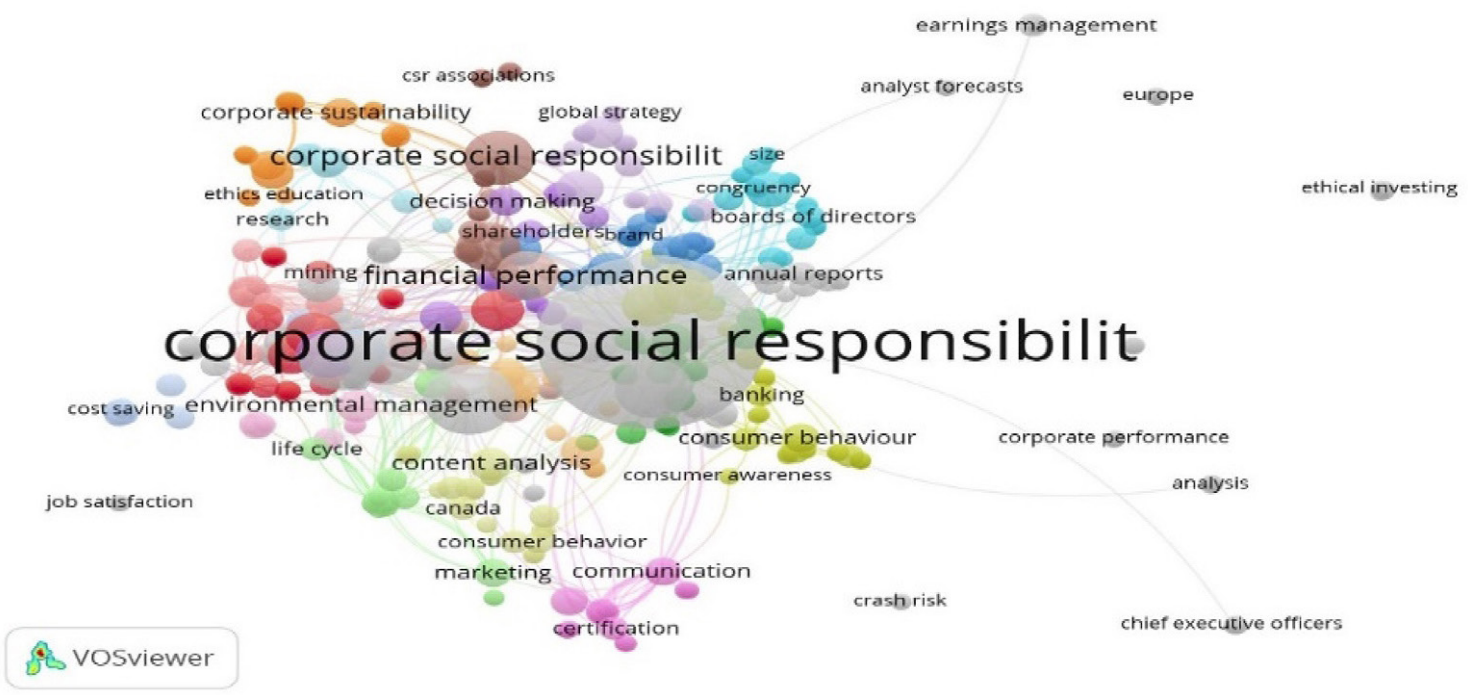

Fuente: Elaboración propia con base en datos de Scopus (2021).

Dentro de la RSU (Figura 3) se visualizan distintas temáticas entra las que destacan aspectos como la sustentabilidad, transparencia, derechos humanos, instituciones de educación superior, impacto social, proceso de toma de decisiones, estudios bibliométricos, calidad ética, entre otros.

\section{Figura 3}

Tópicos abordados sobre la RSU en Scopus, período 1964 - 2020.

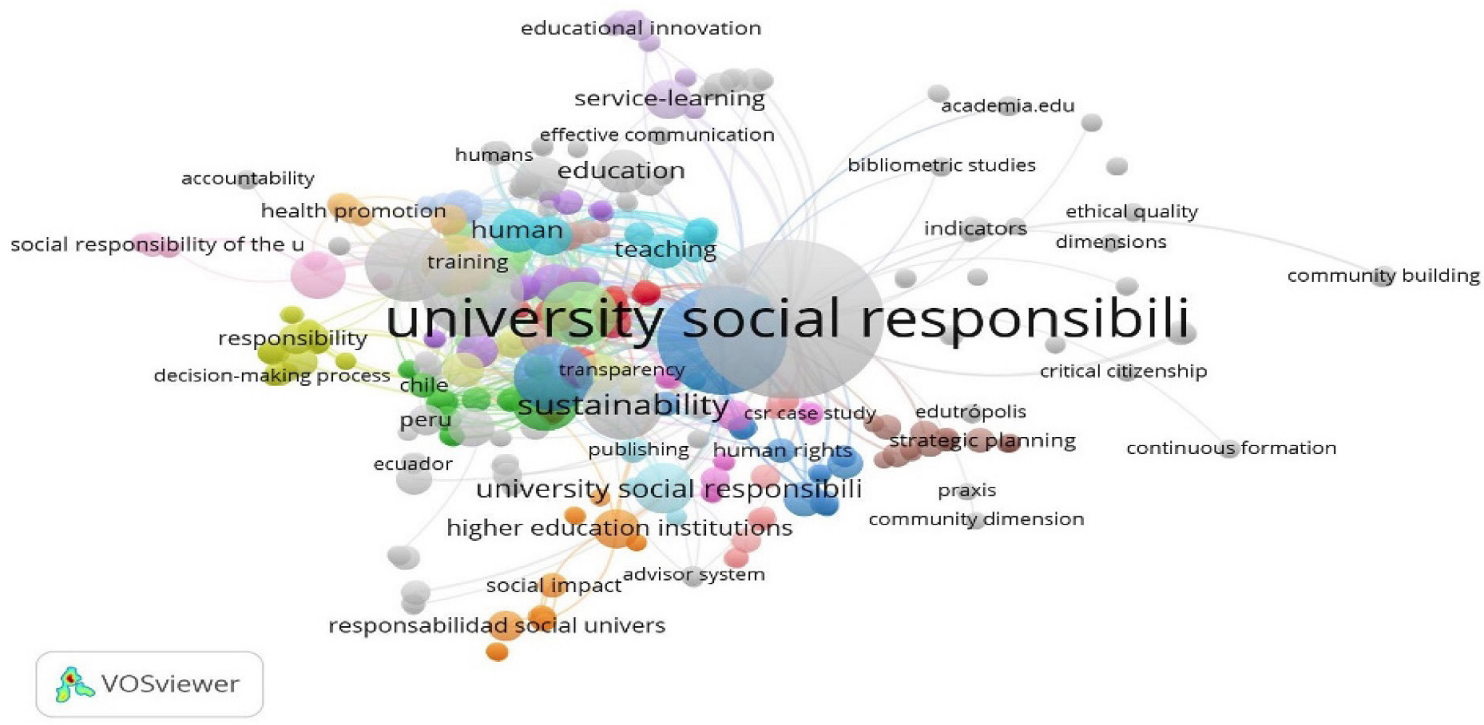

Fuente: Elaboración propia con base en datos de Scopus (2021). 
Dentro de la producción científica analizada correspondiente a la RSI, se pudo constatar que los principales temas que se abordan en este tipo de Responsabilidad Social radican en estudios sobre el ser humano, tomando como factor la pandemia del coronavirus y el proceso de vacunación. Por otro lado, se abordan conceptos como la psicología social y núcleos familiares. En el ámbito de los negocios se aprecian conceptos como el rol de la mujer emprendedora, competición y administración (Figura 4).

\section{Figura 4}

Tópicos abordados sobre la RSI en Scopus, período 1964 - 2020.

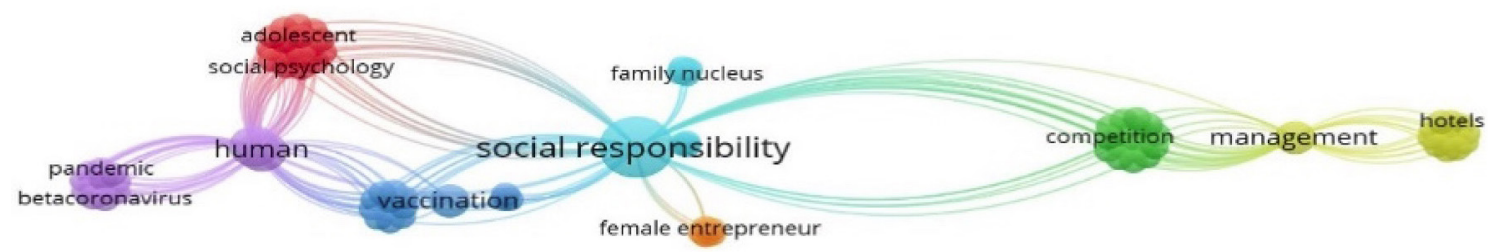

VOSviewer

Fuente: Elaboración propia con base en datos de Scopus (2021).

En lo referente a la RSA se pudo apreciar la relación existente con la RSE, en la que se abordan conceptos como coordinación de canales, costos, comercio, además de estrategias competitivas de Responsabilidad Social Corporativa. Por otra parte, dentro de la producción científica sobre la RSA se abordan aspectos como la medición del desarrollo, impacto medioambiental, estrategia de toma de decisiones, sustentabilidad corporativa, entre otros (Figura 5).

\section{Figura 5}

Tópicos abordados sobre la RSA en Scopus, período 1964 - 2020.

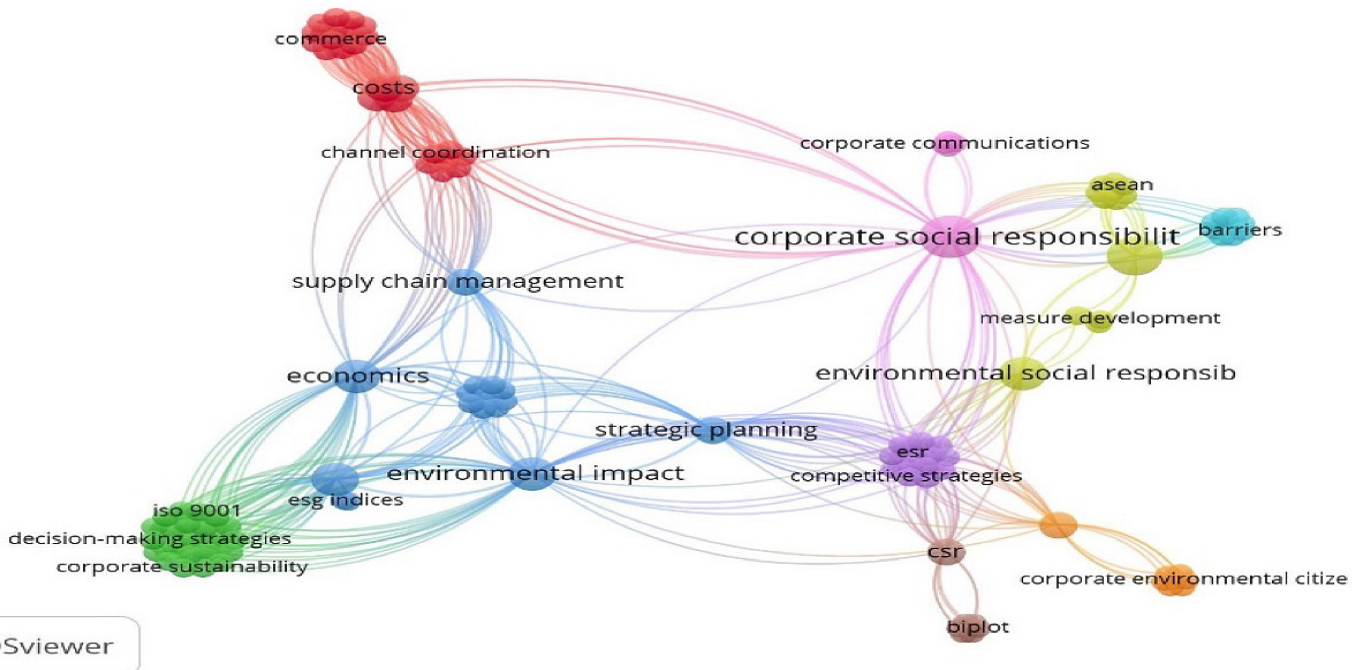

Fuente: Elaboración propia con base en datos de Scopus (2021). 
Finalmente, con relación a la RSG (Figura 6), se apreció que las principales temáticas abordadas en su producción científica tocan temas como la satisfacción de la población en términos de la administración gubernamental, marcos de referencia administrativos, burocracia, rendimiento administrativo, además de factores fiscales políticos, demográficos y socioeconómicos que son determinantes en el contexto de la difusión con respecto a información sobre Responsabilidad Social.

\section{Figura 6}

Tópicos abordados sobre la RSG en Scopus, período 1964 - 2020.

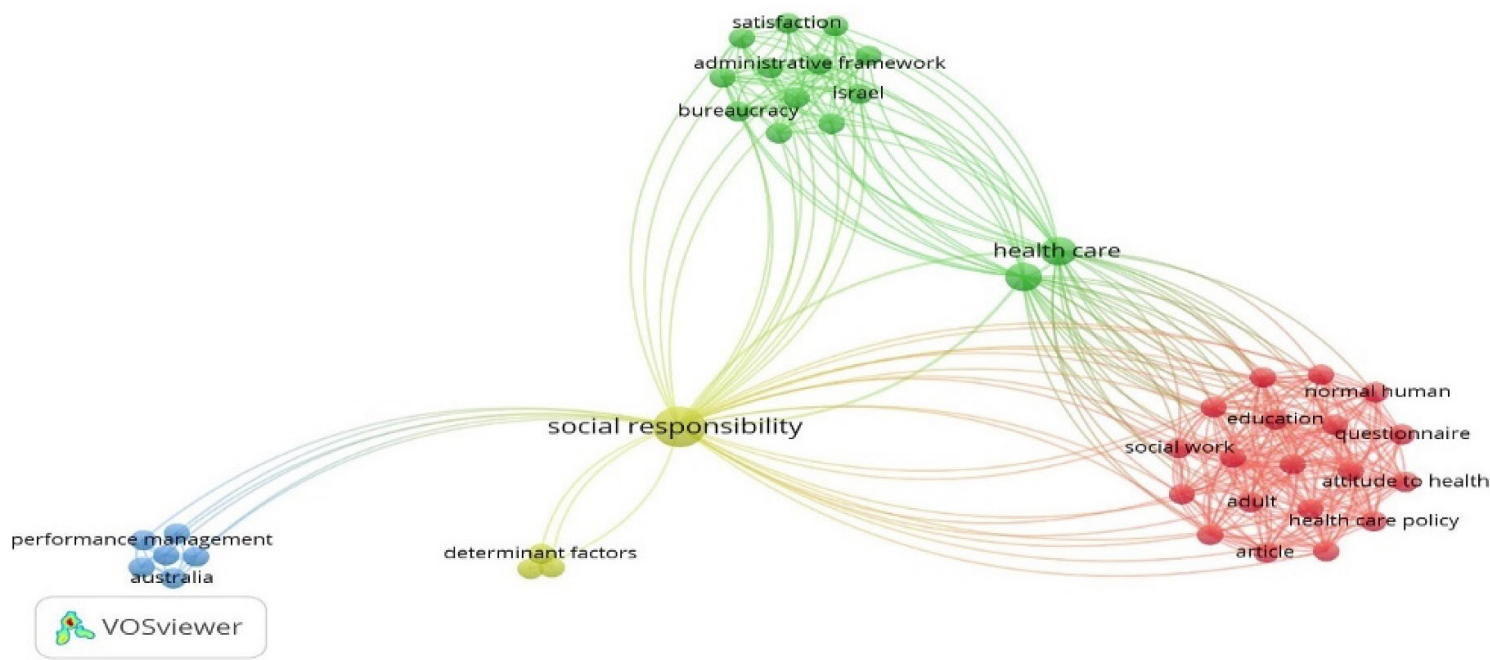

Fuente: Elaboración propia con base en datos de Scopus (2021).

Entre los conceptos que tienen una correlación más fuerte destacan temas como la educación gratuita como una de las principales prioridades para el bienestar social, trabajo social, políticas de salud dirigidas a suplir las necesidades que perciba una comunidad mediante el uso eficiente de los recursos, entre otros temas.

\section{DISCUSIÓN, CONCLUSIONES DE LOS RESULTADOS}

En 56 años de análisis de la producción científica en Responsabilidad Social llama la atención, la lentitud con que se ha posicionado el concepto a nivel de la comunidad científica mundial, en efecto, son casi 39 años de muy baja producción científica, después viene un segundo período en que se quiebra la tendencia donde el tema comienza a interesar y se concluye con una verdadera explosión de productividad que abarca los últimos 17 años, esto tiene su justificación en el reconocimiento expresado por la totalidad de los autores, en relación, a la creciente importancia que tiene la Responsabilidad Social, para el desarrollo integral de la Sociedad.

El análisis bibliométrico reveló que la producción científica está totalmente concentrada en negocios, administración, ciencias sociales, economía y finanzas, de la misma forma, la mayo- 
ría de la producción científica en Responsabilidad Social y sus derivados, está recopilada en 3 revistas Journal Of Business Ethics, Sustainability Switzerland y Corporate Social Responsibility and Environmental Management, esto es muy relevante para el desarrollo científico del área ya que existe un espacio reconocido por la comunidad científica internacional para la divulgación del conocimiento, esto no ocurre de la misma manera en otras áreas vinculada a la administración de organizaciones.

Lo relevante a nivel de la productividad científica está fuertemente enfocado en torno a la Responsabilidad Social Empresarial, dejando en lugares muy menores a las otras categorías de Responsabilidad Social (RSU, RSI, RSA y RSG), en aspectos específicos se pudo constatar que en la Responsabilidad Social Empresarial el tema más recurrente fue el rendimiento financiero, en el caso de la Responsabilidad Social Universitaria lo que acaparó el interés fue la sustentabilidad, en cuanto a la Responsabilidad Social Individual el tratamiento del ser humano como tal lideró las investigaciones, para la Responsabilidad Social Ambiental la coordinación de canales, precios y la gobernanza ambiental en un contexto turístico fueron los más considerados y finalmente en el Responsabilidad Social Gubernamental la satisfacción relacionada al rol que ocupa el gobierno y la confianza ciudadana fue el tema que más se repitió.

Se constató una altísima concentración a nivel de continentes y países involucrados en el desarrollo y la productividad científica relacionado con la Responsabilidad Social, se espera que en futuro cercano esta tendencia se vaya revirtiendo dada la gran relevancia que va tomando el tema a nivel global.

Los niveles de alta concentración se repiten con relación a los tiempos de evolución conceptual, tipos, áreas y revistas, también se replicó a nivel de autores y citaciones, en efecto existe una altísima y muy bien acotada concentración a nivel de autores, en efecto en solo tres de ellos está la gran mayoría del desarrollo científico en Responsabilidad Social Empresarial y a su vez son los que concentran por lejos los mayores niveles de citaciones y co-citaciones.

\section{REFERENCIAS}

Aguinis, H., y A. Glavas. 2012. “What We Know and Don't Know About Corporate Social Responsibility: A Review and Research Agenda". Journal of Management 38(4):932-68.

Anchondo-Granados, Rocío, Javier Tarango, y Gerardo Ascensio-Baca. 2014. “Características de los sujetos-objetos en la producción científica de química avanzada: el caso del CIMAV, México". Ibersid: revista de sistemas de información y documentación 8:143-48.

Arbeláez Gómez, Martha Cecilia, y Javier Onrubia Goñi. 2016. “Análisis bibliométrico y de contenido. Dos metodologías complementarias para el análisis de la revista colombiana Educación y Cultura". Revista de Investigaciones · UCM 14(1):14.

Arnaud, Stéphanie, y David M. Wasieleski. 2014. "Corporate Humanistic Responsibility: Social Performance Through Managerial Discretion of the HRM". Journal of Business Ethics 120(3):313-34.

Bai, Xuan, y Jeanine Chang. 2015. "Corporate social responsibility and firm performance: The mediating role of marketing competence and the moderating role of market environment". Asia Pacific Journal of Management 32(2):505-30. 
De Bakker, F. G. A., P. Groenewegen, y F. Den Hond. 2005. “A Bibliometric Analysis of 30 Years of Research and Theory on Corporate Social Responsibility and Corporate Social Performance". Business and Society 44(3):283-317.

Bandura, A. 2001. "Social Cognitive Theory: An Agentic Perspective". Annual Review of Psychology 52:1-26.

Barbosa Ramírez, David, Christian Medina López, y Myriam Vargas López. 2014. “Globalización, capitalismo financiero y responsabilidad social empresarial: tensiones estructurales". Civilizar Ciencias Sociales y Humanas 14(27):135-54.

Becker, Karen, y Michelle Smidt. 2016. “A risk perspective on human resource management: A review and directions for future research". Human Resource Management Review 26(2):149-65.

Behrend, Joel, y Marc Eulerich. 2019. "The evolution of internal audit research: a bibliometric analysis of published documents (1926-2016)". Accounting History Review 29(1):103-39.

Belter, Christopher W. 2015. "Bibliometric indicators: opportunities and limits". Journal of the Medical Library Association: JMLA 103(4):219-21.

Bernal, Domínguez Deyanira. 2013. “Scopus Sobre Finanzas En a Study in Scopus Bibliometric on Finance in the Period 2004-2011". Teacs 127-40.

Castellanos Domínguez, Óscar Fernando, Aida Mayerly Fúquene Montañez, y Diana Cristina Ramírez Martínez. 2011. Análisis de tendencias: de la información hacia la innovación. Universidad Nacional de Colombia.

Correa Meneses, Juan Sebastián, María del Pilar Rodríguez Córdoba, y Martín Alonso Pantoja Ospina. 2018. "Liderazgo ético en las organizaciones: una revisión de la literatura". $A D$ minister (32):57-82.

Dahlsrud, Alexander. 2008. "How corporate social responsibility is defined: an analysis of 37 definitions". Corporate Social Responsibility and Environmental Management 15(1):1-13.

Donabedian, A. 1988. "The Quality of Care: How Can It Be Assessed?" JAMA: The Journal of the American Medical Association 260(12):1743-48.

Duque Oliva, E. J., A. Cervera Taulet, y C. Rodríguez Romero. 2006. “Estudio bibliométrico de los modelos de medición del concepto de calidad percibida del servicio en Internet". Innovar 16(28):223-43.

Duque, Pedro, y Luis Cervantes. 2019. “Responsabilidad Social Universitaria: una revisión sistemática y análisis bibliométrico". Estudios Gerenciales 35(153):451-64.

Espinoza-Portilla, Elizabeth, Flor Lioo-Jordán, y Gladis J. Villanueva-Cadenas. 2018. “Análisis bibliométrico de las publicaciones peruanas relacionadas a resistencia antimicrobiana en SCOPUS (1992-2017)". Horizonte Médico (Lima) 18(4):75-80.

Fernández, Darcy Luz Mendoza, Marta Cecilia Jaramillo Acosta, y Danny López. 2020. “Responsabilidad social de la Universidad de La Guajira respecto a las comunidades indígenas". Revista de ciencias sociales 26(2):95-106.

Fernández, R. 2009. Responsabilidad Social Corporativa. San Vicente: Editorial Club Universitario.

Franco, S., M. G. Caroli, F. Cappa, y G. Del Chiappa. 2020. “Are You Good Enough? CSR, Quality Management and Corporate Financial Performance in the Hospitality Industry". International Journal of Hospitality Management 88.

Gaede, James, y Ian H. Rowlands. 2018. “Visualizing social acceptance research: A bibliometric review of the social acceptance literature for energy technology and fuels". Energy research E social science 40:142-58.

Hernández Sampieri, Roberto. 2014. Metodología de la investigación. 6a. ed.. editado por C. Fernández Collado y M. del P. Baptista Lucio. México: México : McGraw-Hill. 
Hirsch, J. E. 2005. "An index to quantify an individual's scientific research output". Proceedings of the National Academy of Sciences 102(46):16569-72.

Holman, Luke, Devi Stuart-Fox, y Cindy E. Hauser. 2018. “The gender gap in science: How long until women are equally represented?" PLoS Biology 16(4):1-20.

Houston, David J., y Sybil M. Delevan. 1990. "Public administration research: An assessment of journal publications". Public Administration Review 50(6):674.

Janik, Agnieszka, Adam Ryszko, y Marek Szafraniec. 2020. “Scientific Landscape of Smart and Sustainable Cities Literature: A Bibliometric Analysis". Sustainability 12(3):779.

Javed, M., M. A. Rashid, G. Hussain, y H. Y. Ali. 2020. “The Effects of Corporate Social Responsibility on Corporate Reputation and Firm Financial Performance: Moderating Role of Responsible Leadership". Corporate Social Responsibility and Environmental Management 27(3):1395-1409.

Larrán-Jorge, Manuel, y Francisco-Javier Andrades-Peña. 2015. “Análisis de la responsabilidad social universitaria desde diferentes enfoques teóricos". Revista Iberoamericana de Educación Superior 6(15):91-107.

Legge Jr, Jerome S., y James Devore. 1987. “Measuring productivity in US public administration and public affairs programs 1981-1985". Administration $\mathcal{E}$ Society 19(2):147-56.

Macke, Janaina, y Denise Genari. 2019. "Systematic literature review on sustainable human resource management". Journal of Cleaner Production 208:806-15.

Madero-Gómez, Sergio. 2020. “La satisfacción laboral como efecto moderador entre las prácticas organizacionales de recursos humanos y las líneas estratégicas de responsabilidad social corporativa en México". Estudios Gerenciales 391-401.

Maturana, Diana, y Verónica Andrade. 2019. “La relación entre la formalización de las prácticas de gestión humana y la productividad de las mipymes. Un artículo de revisión". Innovar 29(74):101-14.

Nechi, Salem, Belaid Aouni, y Zouhair Mrabet. 2020. “Managing sustainable development through goal programming model and satisfaction functions". Annals of Operations Research 293(2):747-66.

Pedraza, Norma Angélica. 2020. “El clima y la satisfacción laboral del capital humano: factores diferenciados en organizaciones públicas y privadas". Innovar 30(76):9-24.

Peloza, John, y Jingzhi Shang. 2011. "How can corporate social responsibility activities create value for stakeholders? A systematic review". Journal of the Academy of Marketing Science 39(1):117-35.

Perry, James L., y Kenneth L. Kraemer. 1986. "Research Methodology in the” Public Administration Review," 1975-1984". Public administration review 6(3):215-26.

Pineda Ospina, Diana Lorena. 2015. "Bibliometric analysis for the identification of factors of innovation in the food industry". AD-minister (27):95-126.

Porter, M E, y M. R. Kramer. 2006. “Strategy \& Society: The Link between Competitive Advantage and Corporate Social Responsibility". Harvard Business Review 84(12):78-92.

Porter, Michael E, y Mark R. Kramer. 2006. "Strategy and society". HARVARD BUSINESS REVIEW 84(12):78.

Romero, Xavier A. Vélez, y Evelyn D. Cano Lara. 2016. “Los diferentes tipos de responsabilidad social y sus implicaciones éticas". Dominio de las Ciencias 2(3):117-26.

Rosolen, Talita, y Maria Laura Ferranty Maclennan. 2016. “Strategic human resource management and corporate social responsibility: Evidence from Emerging Markets". Revista Electrónica de Negocios Internacionais 11(2):66-80. 
Saha, Raiswa, Shashi, Roberto Cerchione, Rajwinder Singh, y Richa Dahiya. 2020. “Effect of ethical leadership and corporate social responsibility on firm performance: A systematic review". Corporate Social Responsibility and Environmental Management 27(2):409-29.

Salah, S., A. Rahim, y J. A. Carretero. 2013. “Total Company-Wide Management System: Its Components". International Journal of Business Excellence 6(2):161-91.

Salazar, Alejandra López, José Felipe Ojeda Hidalgo, y Martha Ríos Manríquez. 2017. “La responsabilidad social empresarial desde la percepción del capital humano. Estudio de un caso". Revista de Contabilidad-Spanish Accounting Review 20(1):36-46.

Sánchez Henríquez, Jorge Alejandro, y Ignacio Andres Yáñez Vidal. 2021. “Análisis a la producción científica en auditoría de recursos humanos". CAPIC REVIEW 18(0 SE-Investigación).

Sardana, Deepak, Narain Gupta, Vikas Kumar, y Mile Terziovski. 2020. “CSR 'sustainability' practices and firm performance in an emerging economy". Journal of Cleaner Production 258:120766.

Scimago, Grupo, y Rango Citas. 2006. "El índice h de Hirsch: aportaciones a un debate". El profesional de la información 15(4):304-6.

Severino González, Pedro. 2017. "Responsabilidad social empresarial y conductores de valor: análisis de empresas chilenas que publican informes de sustentabilidad". Multidisciplinary Business Review 10(1):20-34.

Stallings, Robert A., y James M. Ferris. 1988. “Public administration research: Work in PAR, 19401984". Public Administration Review 580-87.

Tello Castrillón, Carlos, y María del Pilar Rodríguez Córdoba. 2014. “Categorías conceptuales para el estudio de la responsabilidad social organizacional". Hallazgos 11(22).

Tomazzoni, Edgar Luis, y Jean Max Tavares. 2014. "Emprendedurismo y gestión de recursos humanos en la hotelería de Brasil: Un estudio bibliométrico". Estudios y perspectivas en turismo 23(3):547-65.

Urdaneta, Mary, María Luisa Cova, Alira Chirinos, y Xiohan González. 2016. “Responsabilidad social en las universidades del municipio Maracaibo del estado Zulia". Revista venezolana de gerencia 21(73):65-85.

Waddock, S. A., y S. B. Graves. 1997. “The Corporate Social Performance-Financial Performance Link". Strategic Management Journal 18(4):303-19.

Weng, Chunhua, Andrew Goldstein, Chi Yuan, y Zhiping Zhou. 2018. "The ranking of scientists". Journal of Biomedical Informatics 79:145-46.

Williamson, Oliver E. 2005. "The economics of governance". American Economic Review 95(2):1-18.

Yang, Nini, Caran Colvin, y Yim-Yu Wong. 2013. "Navigating corporate social responsibility components and strategic options: The IHR perspective". Academy of Strategic Management Journal 12(1):39-58.

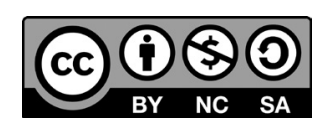

Esta obra está bajo una licencia de

Creative Commons Atribución-NoComercial-CompartirIgual 4.0 Internacional 